\title{
AVALIAÇÃO DA ASSIMETRIA FLUTUANTE EM POPULAÇÕES DE EULAEMA CINGULATA (FABRICIUS, 1804) (HYMENOPTERA: APIDAE) EM DIFERENTES FITOFISIONOMIAS DA MATA ATLÂNTICA
}

\section{$\underline{\text { Lázaro da Silva Carneiro }}$; Cândida Maria Aguiar²; Willian Moura de Aguiar³; Vinina Silva Ferreira ${ }^{4}$}

1. Bolsista PIBIC/CNPq-AF, Graduando em Bacharelado em Ciências Biológicas, Universidade Estadual de Feira de Santana, e-mail: lazarocarneiro16@gmail.com

2. Orientadora, Departamento de Ciências Biológicas, Universidade Estadual de Feira de Santana, e-mail: candida.aguiar@gmail.com

3. Corientador, Instituto do Meio Ambiente e Recursos Hídricos, e-mail: wmag26@yahoo.com.br

4. Corientadora, Universidade Federal do Vale do São Francisco, e-mail: vininasf@gmail.com

PALAVRAS-CHAVE: Assimetria Flutuante; Eulaema nigrita; Fitofisionomias

\section{INTRODUÇÃO}

As abelhas Euglossini (Hymenoptera: Apidae) são importantes polinizadoras, e muitas espécies têm sido utilizadas como bioindicadoras da qualidade do habitat, tal como Eulaema cingulata (F.), que é sugerida como tolerante a áreas abertas e antropizadas (Milet-Pinheiro \& Schlindwein, 2005; Aguiar \& Gaglianone, 2008; 2012).

Uma maneira de mensurar a resposta dos organismos a fatores ambientais é através da assimetria flutuante (FA), que se baseia na diferença entre os lados direito e esquerdo em estruturas bilaterais relacionadas a imprecisões no processo de desenvolvimento ontogenético (Klingenberg, 2015). Aumentos nos níveis de FA estão associados estresses ambientais no desenvolvimento (Klingenberg \& McIntyre, 1998; Klingenberg, 2015).

Embora seja uma importante ferramenta, poucos trabalhos utilizaram a FA para observar como diferentes ambientes influenciam na assimetria de populações de abelhas Euglossini. Silva et al. (2009) compararam os níveis de assimetria flutuante em Euglossa pleosticta Dressler e Eulaema nigrita Lepeletier, provenientes de diferentes ambientes, enquanto Pinto et al. (2015), compararam a assimetria das asas de Eulaema nigrita entre ambientes agrícolas e de cerrado. Assim, são necessários estudos que busquem entender como esses insetos respondem morfologicamente a estresses ao longo de gradientes ambientais. $\mathrm{O}$ objetivo desse estudo foi avaliar os níveis de assimetria flutuante das asas anteriores de Eulaema cingulata de diferentes fitofisionomias da Mata Atlântica.

\section{MATERIAL E MÉTODOS}

Machos de Eulaema cingulata foram amostrados em diferentes fitofisonomias do Corredor Central da Mata Atlântica, Baixo Sul da Bahia, e do Corredor da Serra do Mar, norte do Rio de Janeiro (Tabela 1). Os indivíduos foram coletados utilizando iscas odoríferas e estão depositados no Laboratório de Entomologia- LENT da Universidade Estadual de Feira de Santana- UEFS e no Laboratório de Ciências Ambientais- LCA da Universidade Estadual do Norte Fluminense- UENF.

Tabela 1. Número de indivíduos de Eulaema cingulata (Fabricius) analisados por fitofisionomia da Mata Atlântica, na Área de Proteção Ambiental do Pratigi, Bahia, e norte do 
Rio de Janeiro: FEST: Floresta Estacional Semidecidual de Terras Baixas; FESS: Floresta Estacional Semidecidual Submontana; FODM: Floresta Ombrófila Densa Montana; FODS: Floresta Ombrófila Densa Submontana; CCSE: Cacau-seringa.

\begin{tabular}{cccc}
\hline Fitofisionomia & Município & Coordenadas & $\begin{array}{c}\mathrm{N}^{\circ} \text { de } \\
\text { indivíduos } \\
\text { analisados }\end{array}$ \\
\hline FEST & São Francisco de Itabapoana- RJ & $21^{\circ} 24^{\prime} \mathrm{S} 41^{\circ} 04^{\prime} \mathrm{W}$ & 51 \\
FESS & São José de Ubá-RJ & $21^{\circ} 24^{\prime} \mathrm{S} 42^{\circ} 01^{\prime} \mathrm{W}$ & 47 \\
FODM $_{1}$ & Trajano de Moraes- RJ & $21^{\circ} 48^{\prime} \mathrm{S} 14^{\circ} 40^{\prime} \mathrm{W}$ & 53 \\
FODS & Ibirapitanga- BA & $13^{\circ} 52^{\prime} \mathrm{S} 39^{\circ} 16^{\prime} \mathrm{W}$ & 59 \\
FODM 2 & Ibirapitanga-BA & $13^{\circ} 53^{\prime} \mathrm{S} 39^{\circ} 27^{\prime} \mathrm{W}$ & 78 \\
CCSE & Piraí do Norte-BA & $13^{\circ} 48^{\prime} \mathrm{S} 39^{\circ} 17^{\prime} \mathrm{W}$ & 41 \\
\hline Total & & & 329 \\
\hline
\end{tabular}

As asas anteriores de cada indivíduo foram destacadas, fixadas entre duas lâminas de microscopia e levadas para um estereomicroscópio com câmera acoplada, onde foram fotografadas. Um banco de imagens foi criado no software tpsUtil (Rohlf, 2010), e no programa tpsDig2 (Rohlf, 2008) foram digitalizados 18 landmarks na interseção das nervuras das asas duas vezes, para mensurar o erro de medição.

Os arquivos foram levados para o software MorphoJ (Klingenberg, 2008) para análises estatísticas. Foi realizada uma sobreposição de Procrustes, que remove efeitos não relacionados com a forma. Para acessar a assimetria do tamanho das asas, foi aplicada uma ANOVA two-way, em que o tamanho do centróide é uma variável dependente, lado como efeito fixo e indivíduo como um fator aleatório. A medida de assimetria flutuante é, então, a interação entre essas variáveis (Klingenberg \& McIntyre, 1998; Klingenberg, 2015). Para a forma das asas, utilizou-se a Procrustes ANOVA, que é possível calcular a variação nos níveis de indivíduo, que é a variação entre as médias de ambos os lados de cada indivíduo (Klingenberg \& McIntyre, 1998; Ferreira, 2016), (Klingenberg, 2015), além da interação indivíduo x lado (individuo é o efeito aleatório e lado é o efeito fixo) que serve como uma medida de assimetria flutuante (Klingenberg \& McIntyre, 1998; Klingenberg, 2015).

Para eliminar o efeito da alometria (influência do tamanho na forma), utilizou-se uma análise de regressão multivariada (Klingenberg, 2015). Os componentes simétricos (representa a variação individual) e assimétricos (representa a assimetria flutuante) foram às variáveis dependentes, e o tamanho do centróide a variável independente (Klingenberg et al. 2002; Ferreira, 2016). Juntamente com a análise de regressão multivariada, foi realizado um teste de permutação, com 10000 ciclos, para calcular a significância (Ferreira, 2016; Klingenberg, 2016).

\section{RESULTADOS E DISCUSSÃO}

Para a assimetria do tamanho da asa, a ANOVA two-away indicou diferenças estatisticamente significativas $(p<0.05)$ entre as variáveis lado e interação indivíduo x lado para todas as populações, o que indica a presença de assimetria direcional e flutuante. Para a assimetria da forma da asa, a Procrustes ANOVA indicou diferenças estatisticamente 
significativas $(p<0.05)$ entre os efeitos lado e interação indivíduo x lado, que resulta na ocorrência de assimetria direcional e flutuante.

A assimetria flutuante é apontada como um importante biomarcador para monitorar como estresses ambientais e genéticos interferem sobre o desenvolvimento dos organismos (Klingenberg, 2015), e assim, embora Eulaema cingulata seja indicada como tolerante a diferentes alterações ambientais (Milet-Pinheiro \& Schlindwein, 2005; Aguiar \& Gaglianone, 2012), a presença de assimetria flutuante indica que as populações, independente da fitofisionomia, estão submetidas a diferentes pressões, sejam de origem natural ou antrópica, que podem estar levando a desvios em seu padrão de simetria. Ferreira (2016) também observou a presença de assimetria direcional e FA em Centris analis (Frabicius) e Centris tarsata Smith coletados em ambientes agrícolas e naturais.

A assimetria direcional parece ser geneticamente determinada e adaptativa (Szentgyörgy et al. 2017). Os dados sobre assimetria direcional encontrados no presente estudo reforçam os resultados encontrados por Smith et al. 1997 e Ferreira (2016), que a ocorrência desse tipo de assimetria é comum em abelhas e independe do ambiente analisado, o que pode ser verificado em vários outros estudos com esses insetos (Pinto et al. 2015; Szentgyörgyi et al. 2016).

Os efeitos alométricos para os componentes simétricos (variação individual) e assimétricos (assimetria flutuante) foram pequenos para todas as populações, contudo, a variação individual foi superior à assimetria flutuante em todas as fitofisionomias analisadas (Tabela 2). O maior nível de assimetria flutuante foi observado para a população coletada em FODS, seguido por FESS, FODM 2 , CCSE, FEST e FODM . $_{\text {. }}$

Tabela 2. Variação individual e assimetria flutuante observada nas populações de diferentes fitofisionomias de Mata Atlântica. O valor $P$ refere-se ao teste de Permutação de 10000 ciclos.

\begin{tabular}{|c|c|c|}
\hline Fitofisionomia & Variação individual & Assimetria Flutuante \\
\hline FEST & $7.2 \%, P<0.001$ & $5.1 \%, P<0.001$ \\
\hline FESS & $9.0 \%, P<0.001$ & $2.3 \%, P>0.05$ \\
\hline $\mathrm{FODM}_{1}$ & $9.9 \%, P<0.001$ & $2.6 \%, P>0.05$ \\
\hline FODS & $3.7 \%, P<0.05$ & $1.5 \%, P>0.05$ \\
\hline $\mathrm{FODM}_{2}$ & $4.3 \%, P<0.001$ & $3.3 \%, P<0.001$ \\
\hline CCSE & $5.0 \%, P<0.05$ & $3.3 \%, P>0.05$ \\
\hline
\end{tabular}

Segundo Klingenberg \& McIntyre (1998), é provável que um mesmo processo de desenvolvimento responda diferentemente a fatores genéticos e ambientais, e assim, essa variabilidade na assimetria flutuante observada pode ser consequência de variáveis como flutuação na disponibilidade de recursos entre as estações, fragmentação do habitat, clima, temperatura, além da diversidade genética dessas populações.

\section{CONSIDERAÇÕES FINAIS}

Apesar da tolerância a diferentes condições ambientais, Eulaema cingulata respondeu, através da assimetria da forma e tamanho das asas, a pressões no seu desenvolvimento, o que torna a assimetria flutuante um bom indicador de estresses em abelhas, sendo necessários novos estudos para compreender como diferentes variáveis bióticas e abióticas interferem no desenvolvimento ontogenético desses insetos.

\section{REFERÊNCIAS}

AGUIAR, W.M.; \& GAGLIANONE, M.C. 2008. Comunidade de abelhas Euglossina (Hymenoptera: Apidae) em remanescentes de mata estacional semidecidual sobre tabuleiro no estado do Rio de Janeiro. Neotrop Entomol. 37(2): 118-125. 
AGUIAR, W.M.; \& GAGLIANONE, M.C. 2012. Euglossine bee communities in small forest fragments of the Atlantic Forest, Rio de Janeiro state, southeastern Brazil (Hymenoptera, Apidae). Rev. Bras. de Entomol. 56(2): 210-219.

FERREIRA, V.S. 2016. Análise morfométrica de Centris (Heterocentris) analis (Fabricius, 1804) e Centris (Hemisiella) tarsata Smith, 1874 sob a ótica da biologia reprodutiva. Universidade de São Paulo, Tese.

KLINGENBERG, C.P. 2008. MorphoJ. Faculty of Life Sciences, Universityof Manchester 3: $75-77$.

KLINGENBERG, C.P. 2016. Size, shape, and form: concepts of allometry in geometric morphometrics. Dev Genes Evol. 226(3): 113-137.

KLINGENBERG, C.P.; BARLUENGA, M.; MEYER; A. 2002. Shape analysis of symmetric structures: quantifying variation among individuals and asymmetry. Evolution 56(10): 19091920.

KLINGENBERG, C.P. 2015. Analyzing fluctuating asymmetry with geometric morphometrics: concepts, methods, and applications. Symmetry 7(2): 843-934.

KLINGENBERG, C.P.; \& MCINTYRE, G.S. 1998. Geometric morphometrics of developmental instability: analyzing patterns of fluctuating asymmetry with Procrustes methods. Evolution 52(5): 1363-1375.

MILET-PINHEIRO, P.; \& SCHLINDWEIN, C. 2005. Do euglossine males (Apidae, Euglossini) leave tropical rainforest to collect fragrances in sugarcane monocultures? Rev. Bras. Zool. 22(4): 853-858.

PINTO, N.S.; SILVA, D.P.; RODRIGUES, J.G.; \& DE MARCO, P. 2015. The size but not the symmetry of the wings of Eulaema nigrita Lepeletier (Apidae: Euglossini) is affected by human-disturbed landscapes in the Brazilian Cerrado Savanna. Neotrop Entomol. 44(5):.439447.

ROHLF, F.J. 2008. TpsDIG2. 12. Department of Ecology and Evolution, State University of New York at Stony Brook.

ROHLF, F.J. 2010. tpsUtil version 1.44. Department of Ecology and Evolution, State University of New York at Stony Brook.

SILVA, M.C.; LOMÔNACO, C.; AUGUSTO, S.C.; \& KERR, W.E. 2009. Climatic and anthropic influence on size and fluctuating asymmetry of Euglossine bees (Hymenoptera, Apidae) in a semideciduous seasonal forest reserve. Genet Mol Res. 8(2): 730-737.

SMITH, D.R.; CRESPI, B.J.; \& BOOKSTEIN, F.L. 1997. Fluctuating asymmetry in the honey bee, Apis mellifera: effects of ploidy and hybridization. J Evol Biol. 10(4): 551-574.

SZENTGYÖRGYI, H.; CZEKOŃSKA, K.; \& TOFILSKI, A. 2016. Influence of pollen deprivation on the fore wing asymmetry of honeybee workers and drones. Apidologie 47(5): 653-662.

SZENTGYÖRGYI, H.; MOROŃ, D.; NAWROCKA, A.; TOFILSKI, A.; \& WOYCIECHOWSKI, M. 2017. Forewing structure of the solitary bee Osmia bicornis developing on heavy metal pollution gradient. Ecotoxicology 26(8): 1031-1040. 